\title{
Procedimiento de mejora del proceso de gestión del pedido.
}

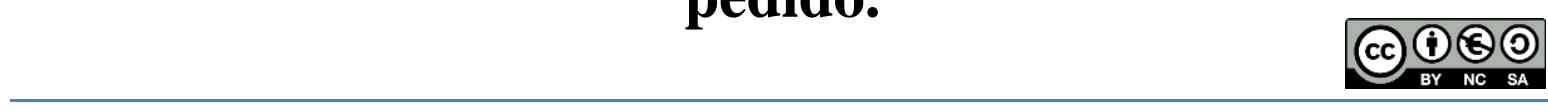

Procedure for improving the process of managing the order.

Alexis Medinilla Sarduy. ${ }^{1}$, Gretel Martínez Curbelo. ${ }^{2}$, Daylí Covas Varela. ${ }^{3}$, Alexander González Yanes. ${ }^{4}$ \& Efraín Velasteguí López. ${ }^{5}$

Recibido: 10-03-2017 / Revisado: 13-05-2017 Aceptado: 09-06-2018/ Publicado: 01-07-2018

\begin{abstract}
.
DOI: https://doi.org/10.33262/cienciadigital.v2i3.142

The customer service is determined by the interaction of multiple factors that in the marketing companies is a function of the order management cycle, so it is essential for them to provide the services at the agreed times and complete them in a period of time appropriate to the Expected by each customer. The present investigation is directed to carry out a procedure that allows a study of the Order Management of the entity object of study, in order to achieve a better organization of this process and in turn a greater satisfaction of the clients. Through the design and implementation of the proposed procedure, an accurate diagnosis was made, which allowed to determine the main problems in the ordering process, as well as its root causes, and the greater dissatisfaction of customers with the service provided by the So that plans for improvements and other solutions are proposed for the most important failures. With the establishment of the proposals it is possible to organize the order cycle and minimize its duration, influencing in this way in the fulfillment of the deadlines and in the elevation of the customer satisfaction.
\end{abstract}

\footnotetext{
1 Universidad de La Habana, Facultad de Turismo, La Habana, Cuba, alexlester.medy@gmail.com 2 Universidad de Cienfuegos, Departamento de Ingeniería Industrial, Facultad de Ciencias Económicas y Empresariales, Cienfuegos, Cuba, alexlester.medy@gmail.com

3 Universidad de Cienfuegos, Departamento de Ingeniería Industrial, Facultad de Ciencias Económicas y Empresariales, Cienfuegos, Cuba, dcovas@ucf.edu.cu

${ }^{4}$ Fábrica de Cemento de Cienfuegos, Cienfuegos, Cuba, Alexander.gonzalez@cementoscfg.cu

5 Universidad Técnica de Cotopaxi Ext la Maná, Cotopaxi, Ecuador, luis.velastegui7838@utc.edu.ec
} 
Keywords: Commercialization; Improvement; Management; Orders; Procedure

\section{Resumen.}

El servicio al cliente queda determinado por la interacción de múltiples factores que en las empresas comercializadoras está en función del ciclo de gestión del pedido, por ello es primordial para ellas prestar los servicios en los tiempos convenidos y completarlos en un período de tiempo adecuado a lo esperado por cada cliente. La presente investigación está dirigida a realizar un procedimiento que permita un estudio de la Gestión de Pedidos de la entidad objeto de estudio, en aras de lograr una mejor organización de este proceso y a su vez una mayor satisfacción de los clientes. A través del diseño e implementación del procedimiento propuesto se logró la realización de un diagnóstico preciso, que permitió determinar los principales problemas existentes en el proceso de pedidos, así como sus causas raíces, y las mayores insatisfacciones de los clientes con el servicio prestado por la empresa, por lo que se proponen planes de mejoras y otras soluciones para los fallos más importantes. Con el establecimiento de las propuestas se logra organizar el ciclo del pedido y minimizar su duración, influyendo de esta forma en el cumplimiento de los plazos de entrega y en la elevación de la satisfacción del cliente.

Palabras Claves: Comercialización; Gestión; Mejora; Pedidos; Procedimiento.

\section{Introducción.}

El turismo es considerado por muchos como la actividad económica más dinámica del siglo $\mathrm{XX}$, es un fenómeno característico e irreversible de nuestra época, con especial importancia en el desarrollo de nuestra sociedad; entre otras causas, por la gran cantidad de personas que en él participan y su amplia repercusión en lo ecológico, económico, social y cultural. A nivel mundial y en el área del Caribe el desarrollo del turismo constituye la principal fuente de ingreso de países en desarrollo. En Cuba es uno de los sectores que más beneficia la reanimación económica por la cantidad de divisa e ingresos que genera y por ello está incluido dentro de las prioridades estratégicas (de Armas, López et al. 2016).

Mantener las empresas con altos niveles competitivos implica una necesidad y obligación de todos aquellos que mantienen una estrecha relación con la actividad. En un mundo caracterizado por la tendencia a la globalización y transnacionalización de la economía los esfuerzos se deben encaminar a la toma de decisiones acertadas. 
En Cuba las empresas turísticas según Daley, Pérez et al. (2016) todavía no han logrado organizar de forma efectiva sus variables y funciones, por lo que no se trabaja con un enfoque integrador para satisfacer al cliente final, de ahí la necesidad de diseñar procesos que perfeccionen el funcionamiento de la distribución en las empresas, facilitando a los directivos la toma de decisiones, una administración más eficiente y que los productos lleguen en tiempo y forma al cliente.

La gestión de pedidos es un proceso que supone un punto de contacto con el cliente. En consecuencias, el éxito del proceso depende de la adecuación del mismo a una amplia variedad de criterios específicos de los clientes, como la facturación personalizada y la facilidad de acceso a la información del pedido. Considerada como parte de la gestión de los clientes se desarrolla a través de actividades que cumplimentan los pedidos a los mismos, a la vez que asegura el máximo valor de la cadena de suministro.

El tiempo requerido para completar las actividades del ciclo del pedido está en el núcleo del servicio al cliente. Se ha estimado que las actividades relacionadas con la preparación, trasmisión, entrada y levantamiento de un pedido representan del 50 al $70 \%$ del tiempo total del ciclo en muchas industrias. Por lo tanto, si se tiene que dar un alto nivel de servicio al cliente mediante tiempos cortos y consistentes con el ciclo del pedido, es esencial que se administren cuidadosamente esas actividades del procesamiento de los pedidos (Ballou 2007).

Es importante para la empresa disponer de un sistema bien definido y organizado en gestión de pedidos del cliente, pues considerarlo como un sistema administrativo de la empresa que únicamente recibe el pedido y lo pasa a producción o a los almacenes para su entrega, es un verdadero error que va degradando la satisfacción de los clientes.

En la implantación de los Lineamientos de la Política Económica del país se manifiesta que en las condiciones actuales resulta un imperativo el incremento de la competitividad de los mercados turísticos, a partir de la elevación de los servicios y el logro de una adecuada coherencia en la relación calidad/precio; además de perfeccionar las formas de comercialización utilizando las tecnologías más avanzadas de la información y las comunicaciones.

Como resultado de la reorientación de la economía cubana y el desarrollo de sectores emergentes que dieran respuesta a las condiciones de cambio del entorno y en la búsqueda de un alto nivel de efectividad, se reinicia en 1980 el desarrollo turístico internacional, manifestándose a un ritmo de crecimiento acelerado lo que lleva a la ampliación de toda una estructura turística que diera respuesta con rapidez y flexibilidad a las exigencias del sector. 
El surgimiento de la Comercializadora ITH, S.A., como casa matriz se inserta dentro de la estructura anteriormente mencionada. La actividad de esta nueva empresa determina un acelerado proceso de su estructura y un crecimiento oportuno de razón económicofinanciero, lo que conlleva a una rápida expansión por el territorio nacional, creándose así la Comercializadora ITH División Cienfuegos.

Esta empresa como otras no está exenta de problemas y deficiencias que impiden un buen desenvolvimiento y logro de sus resultados, por tanto también se involucra en la búsqueda de filosofías que le permitan mejorar sus servicios. Un estudio de la gestión del pedido en la empresa arroja las siguientes situaciones:

- El $63 \%$ de los pedidos en los últimos 6 meses fueron entregados fuera de tiempo causando insatisfacciones en los clientes.

- La dirección de la empresa no está satisfecha con la forma en que los clientes realizan el pedido siendo esta de forma irregular, sin tener en cuenta, la cantidad, el producto o el tiempo entre pedidos.

- Mal aprovechamiento de la capacidad del medio de transporte y del recorrido en la entrega de los pedidos.

- Falta de integración, organización y coordinación entre las actividades de pedido.

Dadas las irregularidades y las insatisfacciones encontradas en el proceso de gestión del pedido de dicha entidad y al no existir una adecuada coordinación e integración entre las actividades de pedidos, es necesario estudiar y mejorar la gestión de estos ciclos eficientemente para lograr un mayor nivel de satisfacción del cliente, por ello la investigación presenta el siguiente problema de investigación: ¿Cómo mejorar la actividad de pedidos en la Empresa Comercializadora Mayorista ITH Cienfuegos?; para dar respuesta a esta problemática se formula como objetivo general: Diseñar un procedimiento para mejorar la Gestión de Pedidos en la Empresa Comercializadora Mayorista ITH Cienfuegos.

\section{Desarrollo.}

\section{Marco Teórico Referencial:}

La gestión de pedidos es un proceso que supone un punto de contacto con el cliente. En consecuencias, el éxito del proceso depende de la adecuación del mismo a una amplia variedad de criterios específicos de los clientes. En este proceso la empresa se relaciona con el cliente de forma sistemática. La experiencia de los clientes con las empresas está fijada por la forma en que se le gestiona el ciclo de vida del pedido: "durante el ciclo de vida del pedido, cada vez que se manipula el pedido, se manipula al cliente. Cada vez que el pedido es desatendido, el cliente se siente desatendido." 
La Gestión de Pedidos encierra las tareas relacionadas a la recepción, aceptación, configuración, manipulación, consulta y conservado del pedido en todas las etapas del ciclo de vida. A pesar de considerarse un tema prioritario en las empresas los requerimientos del mercado vuelven a poner de manifiesto su importancia, destacándose en los trabajos relacionados con el tema su importancia (Valero, Bas et al. 2005).

Las tareas que conforman el proceso de Gestión de Pedidos parten de una correcta identificación y coordinación. De ahí que la inserción de este proceso en diferentes departamentos, empresa y cadenas de suministros pueda traer consigo confusión y ambigüedad, y dificultar una visión objetiva y unificada del mismo (Alemany, Alarcón et al. 2008, MacCarthy and Brabazon 2008). Para Bramham and MacCarthy (2004), las actividades de este proceso se pueden estandarizar para el logro de la eficiencia y el aumento de la satisfacción del cliente, dando lugar incluso a una posterior automatización.

La conjunción de las tareas llevada a cabo en el proceso de gestión de pedidos da lugar a subprocesos que según Shapiro, Rangan et al. (2001) apuntan a 10 actividades básicas en la gestión. En estos estudios según (art congreso) el pedido "front -end" es importante y necesario a tener en cuenta, ya que todas las actividades se iniciarán a partir de él.

Para Bramham and MacCarthy (2004) el sistema "front-end" se define como "el que traduce capacidades organizacionales y servicios en valor para el cliente". Los subprocesos más notables, dentro del proceso de gestión del pedido, desde un punto de vista de las transformaciones del pedido o procesamientos se realizan ahí. Normalmente, desde el "front-end" se activarán los mecanismos necesarios para que el pedido se cumplimente correctamente. Para Makatsoris, Chang et al. (2004), el "front end" de las empresas es lo más cercano al cliente, la conexión de la empresa con el cliente mientras que en el trabajo de Ball, Chen et al. (2004) se define el "front-end" como la parte en contacto con el cliente o parte comercial. Estos autores también indican que las funciones colocadas en el "frontend" dan soporte para responder a las solicitudes del cliente. Estas funciones, colocadas en primer plano, juegan el fundamental papel de vincular los pedidos de los clientes con los recursos de la empresa, de ahí el peso que recibe su estudio.

\section{Marco Metodológico:}

Los servicios turísticos son actividades concebidas para los turistas y que suelen reunir diferentes actividades de entretenimiento y ocio fuera de casa. A criterio de Huertas y Domínguez (2015) para brindar un buen servicio turístico hay que mantener un sistema de aseguramiento y un adecuado diseño del sistema logístico impulsando y buscando nuevas estrategias de gestión que satisfagan una demanda de productos y servicios más eficientes y de mayor valor agregado; teniendo en cuenta el desarrollo de la personalidad del hombre y su exigencia a una mayor calidad de vida. El servicio engloba o se sustenta en un producto y es más abarcador que este. Un producto divorciado del servicio no representa mucho para el cliente y conduce a la enajenación de este (Gómez and Acevedo 2007). 
La empresa Comercializadora ITH Cienfuegos tiene como objetivo fundamental la comercialización de mercancías en el sector del turismo, por lo que es vital para la misma un eficiente proceso de gestión de pedidos. Este proceso en cualquier organización requiere coordinarse de manera que cada elemento actúe bajo la previsión de parámetros claves que caracterizan el funcionamiento de todo el sistema. Es por eso que cada cadena de suministros debe establecer su propio modelo de organización el cual debe responder de forma balanceada a los requerimientos de los clientes.

En la presente investigación se diseña un procedimiento con enfoque de gestión por proceso con el objetivo de analizar y organizar la gestión de pedidos de la Empresa Comercializadora Mayorista ITH Cienfuegos. Para la investigación se realiza una revisión bibliográfica de las distintas fuentes de información que permitieron fundamentar desde los puntos de vista teórico y metodológico los aspectos relacionados con el proceso aprovisionamiento como parte del sistema logístico. Se revisaron los antecedentes y se determinó la evolución de estos conceptos en el transcurso del tiempo.

El procedimiento que se propone en la investigación se articula con una secuencia de tres etapas y pasos a seguir los cuales se muestran a continuación (ver figura 1).

Figura 1. Procedimiento de mejora en la gestión de pedidos. Fuente: Elaboración propia

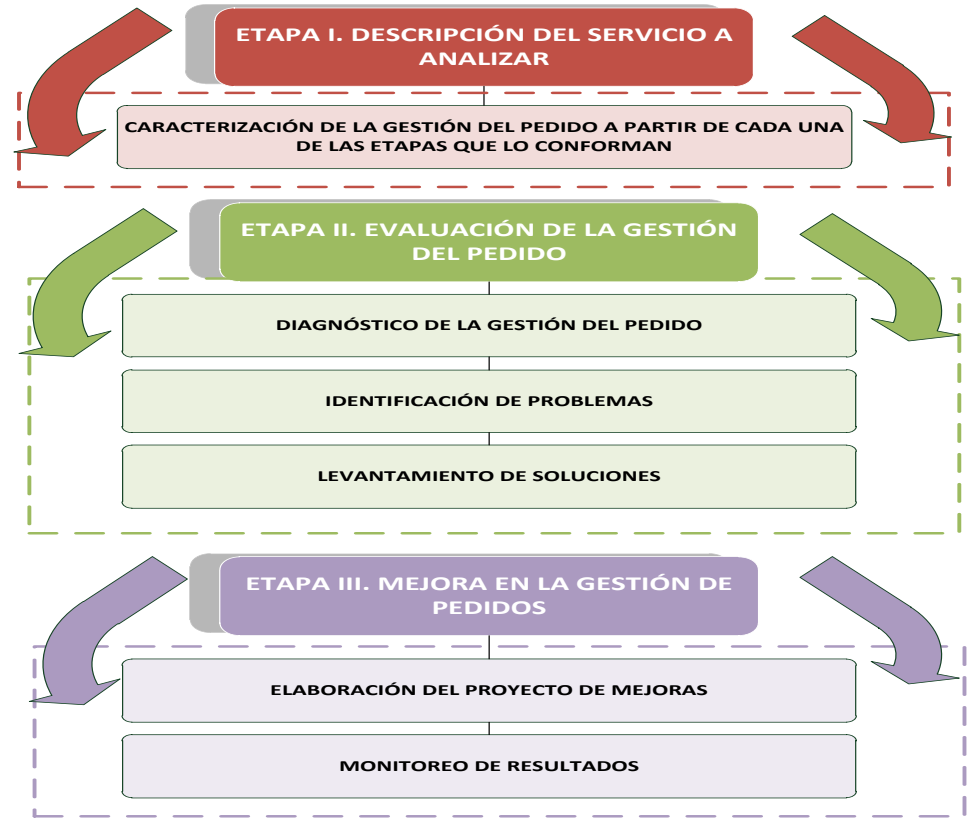

Elaborado por: Grupo de investigación. 
Etapa I: Descripción del servicio a analizar

Paso 1: Caracterización de la Gestión del Pedido a partir de cada una de las etapas que lo conforman.

Etapas de la gestión de pedidos:

- Transmisión del pedido: Esta influye en gran medida sobre la duración del ciclo del cliente, ya que depende directamente del sistema de comunicación cliente-proveedor. La diferencia de tiempo está dada por el método utilizado, donde además de los tradicionales, vía telefónica, correo, etc., se han incorporado con gran fuerza y cada vez con más importancia los métodos electrónicos de transmisión de los pedidos, gracias a la incorporación de los sistemas informáticos en el mundo de la empresa.

- Procesamiento del pedido: Incluye las tareas administrativas, como preparación de facturas, comprobaciones de créditos, gestión del inventario, información a los distintos departamentos de la empresa como producción, marketing, ventas, finanzas, etc. Debe aclararse que en esta etapa debe existir un contacto cliente- empresa donde quede claro los productos a enviar, así como la fecha de entrega. Durante el tratamiento del pedido, puede suceder también, que en la gestión del inventario se detecte que un producto no esté en existencia, por lo que en este caso se puede realizar un pedido hacia el proveedor para cubrir la necesidad.

- Preparación del pedido: Las tareas de preparación del pedido incluyen el despacho de productos, embalaje y consolidación de pedidos, así como la carga para posteriormente ser entregados. En esta etapa juega un papel importante el método de trabajo empleado durante el despacho y la carga, pues de él depende en gran medida el tiempo de esta etapa. Durante la consolidación deben tenerse en cuenta la posibilidad de transportar todos los pedidos que estén en esa misma dirección, para ser entregados.

-Entrega del pedido: La última fase del proceso del pedido es cuando éste es entregado al cliente, de acuerdo a los términos acordado al inicio del proceso.

Posibles herramientas: Revisión y análisis de documentos, entrevista, observación directa, diagramas de flujo.

Etapa II: Evaluación de la gestión de pedidos.

En ella se requiere evaluar el proceso haciendo un estudio minucioso de la actividad en cuanto a su situación actual, los problemas existentes y las alternativas de solución.

Paso 2: Diagnóstico de la Gestión del Pedido: para saber si se cuenta con procesos eficientes de gestión de pedido, y sobre todo, alineados con su estrategia organizacional hay que realizar primero un diagnóstico, el cuál debe permitir hacer una profundización del 
estado actual de la empresa permitiendo luego caracterizar los procesos que la conforman y sus oportunidades de mejora.

Paso 3: Identificación de problemas: la identificación de un problema lleva implícito un profundo y riguroso análisis dirigido a identificar e involucrar los elementos involucrados (elementos que intervienen, parámetros que lo caracterizan y hecho y circunstancias que rodean el problema); estudiar las interrelaciones entre ellos para ayudar en las relaciones de causalidad que permiten explicar la esencia del problema.

Paso 4: Levantamiento de soluciones.

Posibles herramientas: Diagrama de Gantt, lluvia de ideas (Brainstorming), diagrama Causa-Efecto, encuestas a clientes, técnicas de grupo (Método de expertos), análisis de Modo y Efectos de Fallo (FMEA)

Etapa III: Mejoras en la gestión de pedidos.

En esta etapa se pretende planear (elaborar) y monitorear, permanentemente, los cambios para garantizar la calidad del proceso estudiado.

Paso 5: Elaboración del proyecto de mejora.

Paso 6: Monitoreo de resultados.

Posibles herramientas: Plan de mejora 5W y $1 \mathrm{H}$.

\section{Análisis de los Resultados:}

Una vez diseñado el procedimiento a utilizar se valida a través de su implementación en el objeto de estudio; en una primera etapa de la caracterización de las cuatro fases de la gestión del pedido se obtiene:

- La transmisión de los pedidos se realiza vía correo electrónico, llamadas telefónicas, visita personal a la entidad.

- Los pedidos recibidos por las distintas vías de transmisión no tienen procedimientos adecuados para ser tratados de alguna forma específica, todos cuentan con el mismo plazo de entrega, por lo que no existe un volumen mínimo como restricción para aceptar un pedido

- El pedido se trata en el orden en que se recibe, pero tienen prioridad los clientes que realicen su pedido de forma personal y luego se atienden los que utilizaron los métodos de correo o teléfono, solamente se hace la excepción con la Ciénaga de Zapata que realiza su transmisión por correo, ya que por la situación geográfica localizada fuera del territorio se le hace imposible la presencia de alguien para hacer los pedidos, y debido a que es el cliente que más ingresos le aporta a la entidad, tiene a su disposición a un especialista en venta para atender exclusivamente sus solicitudes 
- Para realizar el completamiento del pedido cuando existen productos deficitarios, ITH realiza gestiones de compras con sus proveedores, primeramente hace su pedido a la Industria Nacional, la que le ofrece los productos solo si dispone de un stock de mercancías o de lo contrario a la casa Matriz

- En el caso de que al cliente lleguen los productos defectuosos e inutilizables, se realiza la devolución a ITH que es la encargada de absorber los costos. La empresa cuenta con procedimientos para la devolución y reposición de productos dañados o defectuosos

Un análisis de las actividades que se desarrollan y su coordinación, así como el tiempo de duración de ellas se resume en la Tabla 1, la cual permite realizar la representación del proceso a partir del Modelo general de la organización (MGO) que se muestra en la Figura 2 donde queda reflejada la duración del ciclo del proceso.

Tabla 1. Actividades que conforman el ciclo del pedido.

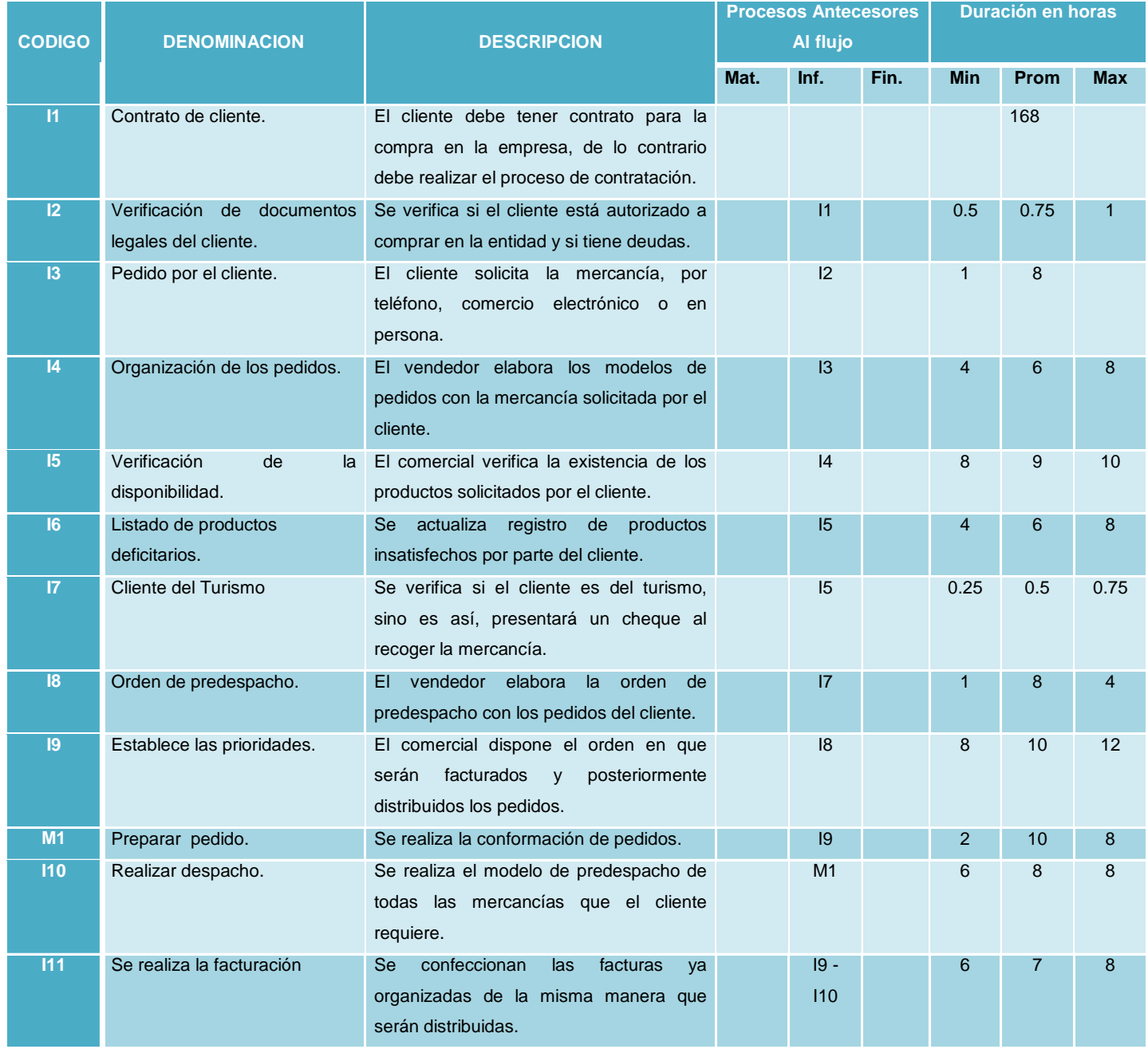

Fuente: Elaboración propia. 
A partir de los resultados obtenidos en los análisis anteriores se puede inferir que el ciclo de pedidos de los clientes del turismo es de aproximadamente 75 horas lo que equivale a 3 horas más de lo establecido en el contrato que son 72 horas.

En el caso de que el cliente utilice los servicios de la empresa por primera vez el tiempo de duración del ciclo es de 243 horas (ver figura 2), pues la actividad de contrato del cliente tiene una duración de 168 horas, lo que equivale a 7 días superior de las 72 horas reguladas.

Figura 2. Tiempo de duración del ciclo para cuando un cliente solicita el servicio por primera vez.

\begin{tabular}{|c|c|c|c|c|c|c|c|c|}
\hline \begin{tabular}{|c|}
$05-30-2013$ \\
$16: 17: 41$
\end{tabular} & $\begin{array}{l}\text { Activity } \\
\text { Name }\end{array}$ & \begin{tabular}{|c|} 
On Critical \\
Path
\end{tabular} & $\begin{array}{c}\text { Activity } \\
\text { Time }\end{array}$ & \begin{tabular}{|c|} 
Earliest \\
Start
\end{tabular} & \begin{tabular}{|c|} 
Earliest \\
Finish
\end{tabular} & \begin{tabular}{|l|} 
Latest \\
Start
\end{tabular} & \begin{tabular}{|l|} 
Latest \\
Finish
\end{tabular} & $\begin{array}{c}\text { Slack } \\
\text { (LS-ES) }\end{array}$ \\
\hline 1 & Contrato con el cliente & Yes & 168 & $\mathbf{0}$ & 168 & $\mathbf{0}$ & 168 & $\mathbf{0}$ \\
\hline 2 & Verificación de documentos & Yes & 0.75 & 168 & 168.75 & 168 & 168.75 & $\mathbf{0}$ \\
\hline 3 & Pedido & Yes & 8 & 168.75 & 176.75 & 168.75 & 176.75 & $\mathbf{0}$ \\
\hline 4 & Organización de pedido & Yes & 6 & 176,75 & 182,75 & 176,75 & 182.75 & $\mathbf{0}$ \\
\hline 5 & Verificación de disponibilidad & Yes & 9 & 182,75 & 191,75 & 182,75 & 191.75 & $\mathbf{0}$ \\
\hline 6 & Listado de productos & no & 6 & 191.75 & 197.75 & 237.87 & 243.87 & 46.12 \\
\hline 7 & Clientes del turismo & Yes & 5 & 191.75 & 196,75 & 191,75 & 196.75 & $\mathbf{0}$ \\
\hline 8 & Orden de predespacho & Yes & 8 & 196.75 & 204.75 & 196,75 & 204.75 & $\mathbf{0}$ \\
\hline 9 & Establecimiento de prioridades & Yes & 10 & 204.75 & 214.75 & 204,75 & 214.75 & $\mathbf{0}$ \\
\hline 10 & Prepara pedido & Yes & 10 & 214.75 & 224,75 & 214,75 & 224,75 & $\mathbf{0}$ \\
\hline 11 & Realizar despacho & no & 8 & 224.75 & 232,75 & 235,87 & 243.87 & 11.12 \\
\hline 12 & Facturación & Yes & 7 & 224.75 & 231.75 & 224.75 & 231.75 & $\mathbf{0}$ \\
\hline 13 & Asignación de transporte & Yes & 4 & 231.75 & 235,75 & 231,75 & 235.75 & $\mathbf{0}$ \\
\hline 14 & Carga al trasnporte & Yes & 6 & 235.75 & 241.75 & 235,75 & 241,75 & $\mathbf{0}$ \\
\hline \multirow[t]{3}{*}{15} & Entrega al cliente & Yes & 2,12 & 241,75 & 243.87 & 241,75 & 243.87 & $\mathbf{0}$ \\
\hline & Project & Completion & Time & $=$ & 243.87 & horas & & \\
\hline & Number of & Critical & Path[s] & $=$ & 2 & & & \\
\hline
\end{tabular}

Elaborado por: Grupo de investigación.

Etapa II. Evaluación de la gestión del pedido.

Paso 2. Diagnóstico de la gestión del pedido

Para diagnosticar la gestión del pedido se diseña una encuesta de satisfacción que es aplicada a una muestra de 22 clientes tomándose los más significativos de la empresa; la misma se valida a partir de diferentes criterios que se muestran en la Tabla 2. En todos los casos los resultados obtenidos son positivos y muestran que la encuesta es válida. 
Tabla 2. Criterios para la validación de la encuesta.

\begin{tabular}{cc}
\hline Criterios para la validación de la encuesta & Valor \\
\hline Alpha de Cronbach & 0,710 \\
Medida de adecuación muestral de Kaiser-Meyer-Olkin & 0,551 \\
\hline
\end{tabular}

Fuente: Elaboración propia.

Una vez aplicada la encuesta se obtienen los resultados de cada uno de sus indicadores (mostrándose en la Tabla 3), de la que se puede concluir que las principales insatisfacciones de los clientes las cuales están dadas en:

- Cumplimiento con los plazos de entrega.

- Cumplimiento de la oferta.

- Estabilidad en los suministros.

- Productos sustitutos en caso de carencias.

Tabla 3. Resultados de los indicadores de la encuesta.

\begin{tabular}{|l|l|}
\hline & \\
\hline El transporte utilizado para la entrega de los pedidos es el adecuado & Adecuado $100 \%$ \\
\hline Cumplimiento con los plazos de entrega & No adecuado $100 \%$ \\
\hline Respuesta ante problemas o reclamaciones & Adecuado $100 \%$ \\
\hline Conformidad con el horario establecido. & Adecuado $100 \%$ \\
\hline Calidad de los productos que se ofertan. & Adecuado $100 \%$ \\
\hline Cumplimiento de la oferta. & No Adecuado 90,9\% \\
\hline Estabilidad en los suministros. & No Adecuado $77,3 \%$ \\
\hline Errores en la entrega. & Adecuado $90,9 \%$ \\
\hline Se ofrecen productos sustitutos en caso de carencias. & No Adecuado $86,4 \%$ \\
\hline Habilidades y conocimientos de los empleados. & Adecuado $90 \%$ \\
\hline Trato del personal que interactúa con usted. & Adecuado $90,9 \%$ \\
\hline Información a los clientes sobre la situación de sus pedidos. & Adecuado $90,9 \%$ \\
\hline Se ofrece un servicio que vale lo que el cliente paga por él. & Adecuado $90,9 \%$ \\
\hline $\begin{array}{l}\text { Los precios son más competitivos en comparación al resto de las demás } \\
\text { comercializadoras. }\end{array}$ & Adecuado 90,9\% \\
\hline
\end{tabular}

Elaborado por: Grupo de investigación.

Los resultados de la encuesta son utilizados como base en una sesión con expertos, con el propósito de obtener las causas probables que pueden estar incidiendo en el proceso. A 
partir de este paso se reflejan todas las ideas en un diagrama Causa - Efecto utilizando la técnica de los cinco ¿por qué? donde se logra visualizar hacia donde están asociados un grupo de problemas y diagnosticar sus posibles causas. Establecidas las posibles causas se decide realizar un Análisis de Modo y Efectos de Fallo (FMEA).

Los fallos potenciales considerados en la aplicación de esta técnica son los problemas reales que se obtienen a partir de un trabajo con expertos los resultados de la aplicación de esta técnica se muestra en la Tabla 4.

Tabla 4. Análisis de Modo y Efecto de Fallos.

\begin{tabular}{|c|c|c|c|c|c|c|c|c|}
\hline No. & Fallo & Efecto & Sev & Causas & OOC & Control & EDT & RPM \\
\hline \multirow[t]{3}{*}{1} & \multirow[t]{3}{*}{$\begin{array}{l}\text { Insuficiente } \\
\text { disponibilidad de medios }\end{array}$} & \multirow[t]{3}{*}{$\begin{array}{l}\text { Atraso en la entrega de } \\
\text { pedidos }\end{array}$} & 4 & $\begin{array}{l}\text { Mala planificación del } \\
\text { transporte }\end{array}$ & 6 & $\begin{array}{l}\text { Estudiar una correcta } \\
\text { planificación del } \\
\text { transporte }\end{array}$ & 5 & 120 \\
\hline & & & 3 & $\begin{array}{l}\text { Medios de transportes } \\
\text { rotos }\end{array}$ & 4 & $\begin{array}{l}\text { Plan de Mantenimiento } \\
\text { Preventivo Planificado }\end{array}$ & 3 & 36 \\
\hline & & & 2 & $\begin{array}{l}\text { Medios de transporte en } \\
\text { mantenimiento }\end{array}$ & 4 & $\begin{array}{l}\text { Plan de Mantenimiento } \\
\text { Preventivo Planificado }\end{array}$ & 3 & 24 \\
\hline \multirow[t]{2}{*}{2} & \multirow[t]{2}{*}{$\begin{array}{l}\text { Malas condiciones de } \\
\text { almacenamiento }\end{array}$} & \multirow[t]{2}{*}{$\begin{array}{l}\text { Mala calidad del producto } \\
\text { almacenado }\end{array}$} & 2 & $\begin{array}{l}\text { Insuficientes medios de } \\
\text { almacenamiento }\end{array}$ & 4 & $\begin{array}{l}\text { Planificación de la } \\
\text { cantidad de medios } \\
\text { unitarizadores } \\
\text { necesarios }\end{array}$ & 2 & 24 \\
\hline & & & 3 & $\begin{array}{ll}\text { Incorrecta utilización } & \text { de } \\
\text { los medios } & \text { de } \\
\text { almacenamiento } & \end{array}$ & 5 & $\begin{array}{l}\text { Estudio de selección } \\
\text { de los medios } \\
\text { unitarizadores } \\
\text { adecuados según la } \\
\text { carga }\end{array}$ & 3 & 45 \\
\hline \multirow[t]{2}{*}{3} & \multirow[t]{2}{*}{$\begin{array}{l}\text { Mala organización de los } \\
\text { productos a almacenar }\end{array}$} & \multirow[t]{2}{*}{ Productos fuera de lugar } & 3 & $\begin{array}{l}\text { Insuficiente capacidad de } \\
\text { almacén }\end{array}$ & 4 & $\begin{array}{l}\text { Balance Demanda- } \\
\text { Capacidad }\end{array}$ & 2 & 24 \\
\hline & & & 3 & $\begin{array}{l}\text { Mala utilización del área y } \\
\text { volumen del almacén en } \\
\text { la organización de los } \\
\text { productos }\end{array}$ & 5 & $\begin{array}{l}\text { Estudio de Distribución } \\
\text { en Planta del Almacén }\end{array}$ & 2 & 30 \\
\hline \multirow[t]{2}{*}{4} & \multirow[t]{2}{*}{$\begin{array}{l}\text { Demora en el despacho } \\
\text { de los productos }\end{array}$} & \multirow[t]{2}{*}{$\begin{array}{l}\text { Atraso en la entrega de } \\
\text { pedidos }\end{array}$} & 4 & \multirow[t]{2}{*}{$\begin{array}{l}\text { El almacenero realiza } \\
\text { recorridos innecesarios }\end{array}$} & 6 & $\begin{array}{l}\text { Estudio de Métodos de } \\
\text { trabajo }\end{array}$ & 3 & 72 \\
\hline & & & 4 & & 6 & $\begin{array}{l}\text { Estudio de Medios de } \\
\text { Manipulación Internos } \\
\text { según las cargas }\end{array}$ & 2 & 48 \\
\hline 5 & $\begin{array}{l}\text { Mala organización en la } \\
\text { tramitación de pedidos }\end{array}$ & $\begin{array}{l}\text { Quejas de los clientes por } \\
\text { atrasos en los pedidos }\end{array}$ & & $\begin{array}{l}\text { No establecimiento de } \\
\text { prioridades en el } \\
\text { tratamiento de los pedidos }\end{array}$ & 4 & $\begin{array}{l}\text { Establecer criterios de } \\
\text { prioridades en el } \\
\text { tratamiento del pedido }\end{array}$ & 2 & 10 \\
\hline \multirow[t]{2}{*}{6} & \multirow[t]{2}{*}{ Mala gestión de compras } & \multirow[t]{2}{*}{$\begin{array}{l}\text { Débil y vulnerable sistema } \\
\text { de inventario }\end{array}$} & 8 & $\begin{array}{l}\text { No se trabaja con la } \\
\text { demanda real de los } \\
\text { clientes }\end{array}$ & 10 & $\begin{array}{l}\text { Concientizar al cliente } \\
\text { de establecer un } \\
\text { adecuado sistema de } \\
\text { planificación de la } \\
\text { demanda }\end{array}$ & 8 & 640 \\
\hline & & & 3 & $\begin{array}{l}\text { Impera un Sistema de } \\
\text { abastecimiento push }\end{array}$ & 5 & $\begin{array}{l}\text { Implantar un Sistema } \\
\text { de abastecimiento pull } \\
\text { donde el cliente es } \\
\text { quien hala el } \\
\text { inventario }\end{array}$ & 3 & 45 \\
\hline 7 & Inestabilidad en & El cliente no encuentra los & 4 & gestión & 7 & Sistema & 3 & 84 \\
\hline
\end{tabular}




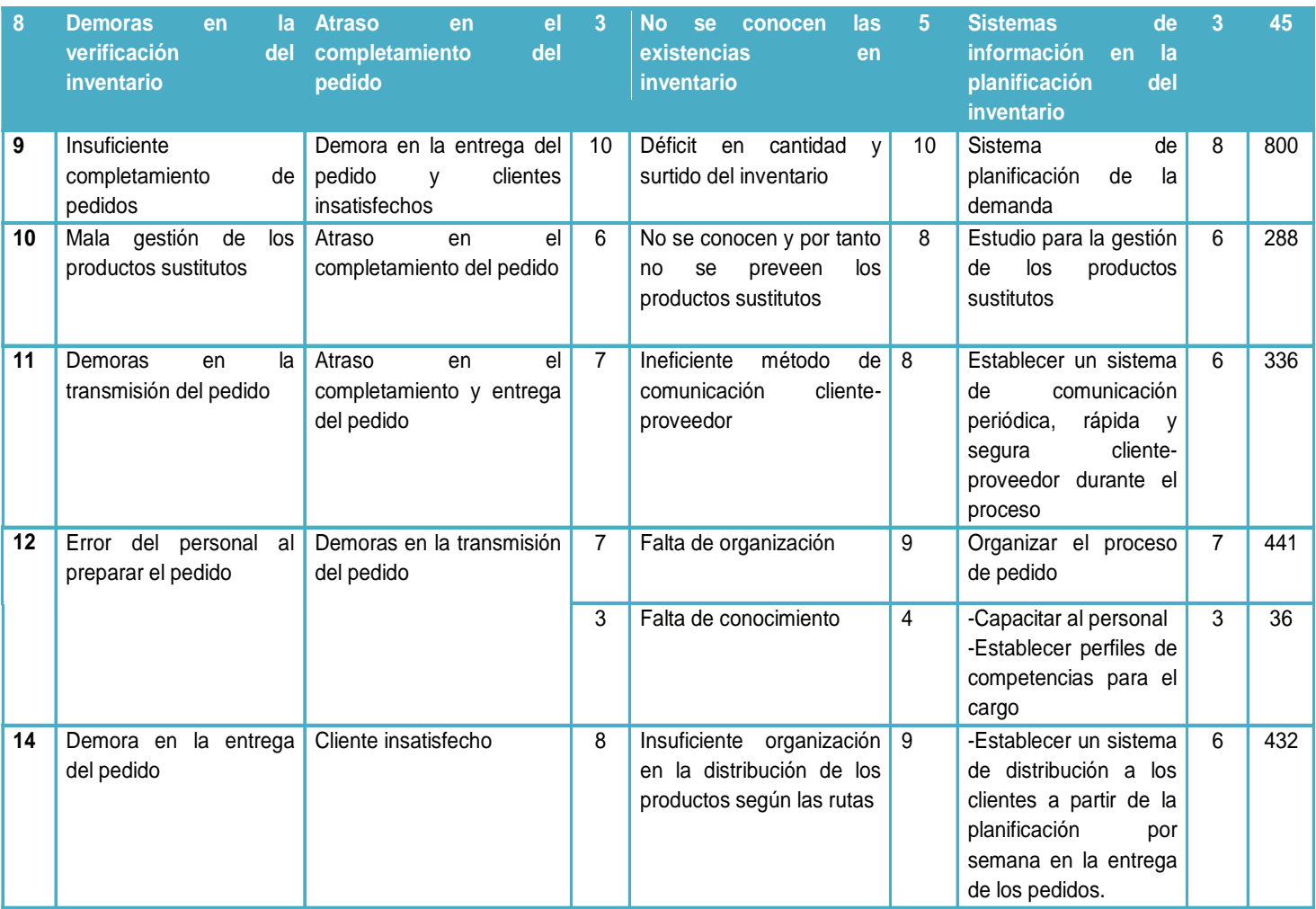

Elaborado por: Grupo de investigación.

Paso 3. Identificación de problemas.

Con la obtención de los principales problemas se detectan las fallas potenciales existentes en el proceso de gestión de pedidos; los mismos se reflejan en un diagrama de interrelaciones con el fin de establecer las relaciones que existen entre las prioridades de mejora detectadas de los fallos potenciales del proceso y las insatisfacciones de los clientes (ver Gráfico 1), para así recurrir a la identificación de las acciones que podrían eliminar o reducir la posibilidad de ocurrencia del fallo potencial y documentar el proceso.

El FMEA juega un papel fundamental en la identificación de los fallos antes de que ocurran posibilitando con ello la ejecución de acciones preventivas. 
Gráfico 1. Diagrama de interrelaciones.

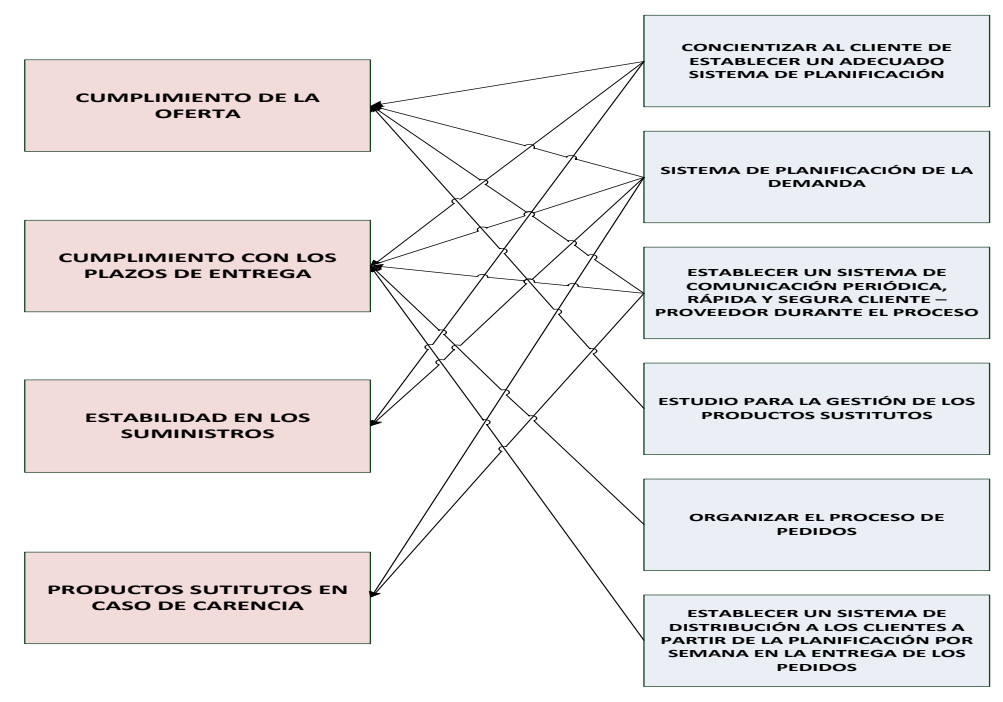

Gráfico 1. Diagrama de interrelaciones.

Del análisis realizado se puede concluir que existe una estrecha relación entre las insatisfacciones en el proceso de Gestión de Pedido presentadas por los clientes de la Comercializadora ITH y los fallos potenciales obtenido a través del análisis de Modos y Efectos de Fallo.

Paso 4: Levantamiento de soluciones.

Los mayores problemas del proceso inciden en la satisfacción de los clientes en cuanto al cumplimiento de los plazos de entrega y en el cumplimiento de la oferta, siendo la mala planificación de la demanda por parte de los clientes la causa que más incide en las insatisfacciones detectadas. Por lo que se deduce que las prioridades a establecer en las oportunidades de mejora para la empresa se muestran de la manera siguiente:

- Sistema de planificación de la demanda

- Concientizar al cliente de establecer un adecuado sistema de planificación de la demanda

- Organizar el proceso de pedido

- Establecer un sistema de distribución a los clientes a partir de la planificación por semana en la entrega de los pedidos

- Establecer un sistema de comunicación periódica, rápida y segura cliente-proveedor durante el proceso

- Estudio para la gestión de los productos sustitutos.

Etapa III. Mejora en la Gestión del pedido

Paso 5. Elaboración del proyecto de mejora 
En el análisis de las propuestas de soluciones se establecen las acciones a desarrollar para la implementación de estas prioridades de mejora a partir de planes elaborados para cada oportunidad.

El establecimiento de las mismas posibilitará que el ciclo del pedido quede más organizado y la tendencia es a disminuir su duración influyendo de esta forma en la elevación de la satisfacción del cliente y en el cumplimiento de los tiempos de entrega.

Paso 6. Monitoreo de los resultados

Para monitorear los resultados del proceso y medir la eficacia del mismo se proponen indicadores de pedido perfecto, como lo indica la tabla que se muestra a continuación.

Tabla 5. Indicadores de pedidos.

\begin{tabular}{|c|c|c|}
\hline Indicador & Descripción & Expresión de cálculo \\
\hline Calidad de los Pedidos Generados & $\begin{array}{c}\text { Pedido sin deterioro ni daño } \\
\text { físico }\end{array}$ & $\begin{array}{c}\text { Pedidos entregados sin } \\
\text { problemas / Total de pedidos } \\
\text { generados } \mathrm{x} 100\end{array}$ \\
\hline Calidad de los Pedidos Generados & $\begin{array}{l}\text { Documentación completa y } \\
\text { correcta }\end{array}$ & $\begin{array}{c}\text { Pedidos entregados sin } \\
\text { problemas / Total de pedidos } \\
\text { generados } \mathrm{x} 100\end{array}$ \\
\hline $\begin{array}{l}\text { Nivel de cumplimiento de los } \\
\text { pedidos }\end{array}$ & $\begin{array}{l}\text { Pedidos entregados en la } \\
\text { Fecha prevista. }\end{array}$ & $\begin{array}{l}\text { Pedidos entregados en } \\
\text { tiempo / Total Pedidos } \\
\text { entregados x } 100\end{array}$ \\
\hline $\begin{array}{l}\text { Nivel de cumplimiento de los } \\
\text { pedidos }\end{array}$ & $\begin{array}{l}\text { Pedidos entregados completos } \\
\text { en cantidad. }\end{array}$ & $\begin{array}{c}\text { Pedidos entregados } \\
\text { completos/ total de pedidos } \\
\text { entregados x } 100\end{array}$ \\
\hline
\end{tabular}

Fuente: Rodríguez, 2010.

Para el cálculo de estos indicadores la empresa debe garantizar la recogida de información a partir de los documentos de trabajo, que faciliten los datos necesarios, así como el sistema de facturación comercial. De igual manera hay que tenerlo en cuenta para cualquier cambio a este sistema, de manera que permita extraer esta información, como medida de control.

\section{Conclusiones.}

- La aplicación correcta del procedimiento propuesto con enfoque de gestión por proceso permitió estudiar, evaluar y proponer el conjunto de acciones que favorecen el proceso de gestión del pedido de la Empresa Comercializadora Mayorista ITH Cienfuegos.

- La encuesta aplicada a los clientes así como las técnicas empleadas en el estudio para caracterizar y diagnosticar el proceso muestran las 
insatisfacciones de estos con respecto al cumplimiento en los plazos de entrega y de la oferta, la estabilidad en los suministros y la carencia de productos sustitutos; los problemas detectados están fundamentalmente asociados a las dos primeras causas de las insatisfacciones de los clientes.

- Las prioridades de mejora estuvieron orientadas a concientizar al cliente de establecer un adecuado sistema de planificación de la demanda, organizar la actividad de pedidos en cuanto a distribuir por días los pedidos de los clientes y establecer un sistema de distribución a los clientes a partir de la planificación en la entrega fundamentalmente.

- El indicador de pedido perfecto que se le propone a la empresa le permitirá monitorizar el comportamiento de la gestión del pedido para cada uno de sus clientes y de forma general para la empresa.

\section{Referencias bibliográficas.}

Alemany, M., et al. (2008). "Order promising process for extended collaborative selling chain." Production Planning and Control 19(2): 105-131.

Ball, M. O., et al. (2004). Available to promise. Handbook of quantitative supply chain analysis, Springer: 447-483.

Ballou, R. H. (2007). Business logistics/supply chain management, 5/E (With Cd), Pearson Education India.

Bramham, J. and B. MacCarthy (2004). "The demand driven chain [product configurator]." Manufacturing Engineer 83(3): 30-33.

Daley, D. S., et al. (2016). "DISEÑO PARA LA OPTIMIZACIÓN DEL PROCESO DE DISTRIBUCIÓN Y TRANSPORTE DEL GRUPO EMPRESARIAL COMERCIAL CARACOL DEL TURISMO." Universidad\&Ciencia 4(3): 1-16.

de Armas, R. L. F., et al. (2016). "El turismo: fuente de financiamiento para el desarrollo en Cuba/The tourism: a source of incomes for the economic development of Cuba." Retos Turísticos 15(2).

Gómez, M. and J. Acevedo (2007). La Logística Moderna en la Empresa, vol. 1, La Habana, LOGICUBA, 2007, ISBN 978-959-7191-17-9.

MacCarthy, B. L. and P. Brabazon (2008). "Order fulfillment in high variety production environments." Journal of Intelligent Manufacturing. 
Makatsoris, H., et al. (2004). "Design of a distributed order promising system and environment for a globally dispersed supply chain." International Journal of Computer Integrated Manufacturing 17(8): 679-691.

Shapiro, B. P., et al. (2001). "Staple yourself to an order." Understanding Business: Processes: 187.

Valero, F. A., et al. (2005). "Order promising” y Gestión de Pedidos: una visión de procesos. IX Congreso de Ingeniería de Organización. 
Para citar el artículo indexado.

Medinilla A., Martínez G., Covas D. \& González A. Velasteguí E. (2018). Procedimiento de mejora del proceso de gestión del pedido. Revista electrónica Ciencia Digital 2(3), 130147.

http://cienciadigital.org/revistacienciadigital2/index.php/CienciaDigital/article/view/142/12 $\underline{7}$

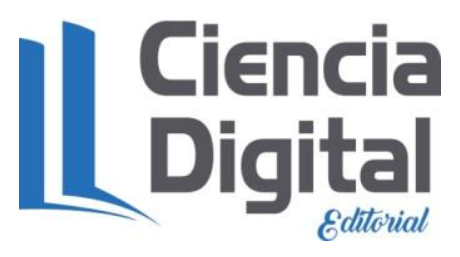

El artículo que se publica es de exclusiva responsabilidad de los autores y no necesariamente reflejan el pensamiento de la Revista Ciencia Digital.

El articulo queda en propiedad de la revista y, por tanto, su publicación parcial y/o total en otro medio tiene que ser autorizado por el director de la Revista Ciencia Digital.
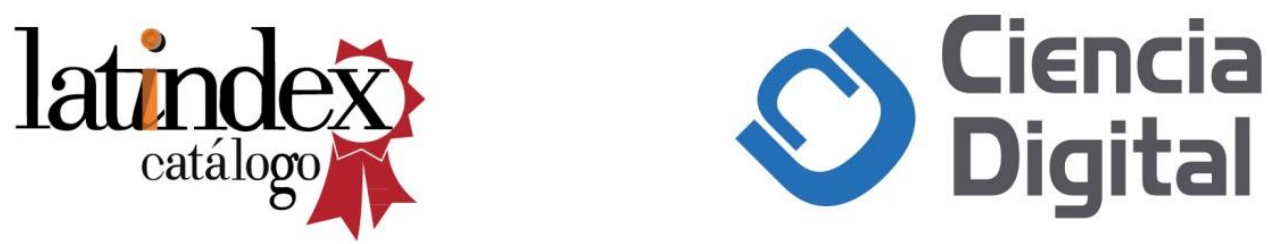